msh-mss Mathématiques et sciences humaines

160 | Hiver 2002

Varia

\title{
"Mathématiques et Sciences humaines" a quarante
} ans

"Mathematics and Social Sciences" is forty years old

Marc Barbut

\section{CpenEdition}

1 Journals

Édition électronique

URL : http://journals.openedition.org/msh/2914

DOI : $10.4000 / \mathrm{msh} .2914$

ISSN : 1950-6821

Éditeur

Centre d'analyse et de mathématique sociales de l'EHESS

Édition imprimée

Date de publication : 1 décembre 2002

ISSN : 0987-6936

Référence électronique

Marc Barbut, " "Mathématiques et Sciences humaines" a quarante ans », Mathématiques et sciences humaines [En ligne], 160 | Hiver 2002, mis en ligne le 10 février 2006, consulté le 23 juillet 2020. URL: http://journals.openedition.org/msh/2914 
Math. \& Sci. hum., (40e année, ${ }^{\circ} 160,2002$, p. 5-6)

\section{MATHÉMATIQUES ET SCIENCES HUMAINES A QUARANTE ANS}

C'est en effet en novembre 1962 que fut publié le premier numéro, très précisément le 22 de ce mois!; le prix de l'abonnement annuel, pour quatre numéros, n'était que de 20 francs (on disait encore «!nouveaux francs!») de l'époque!!

La décision de créer ce périodique avait été prise six mois auparavant. Voici dans quelles circonstances.

Du 24 au 26 mai 1962, s'est tenu à Paris, dans les locaux du Laboratoire d'Anthropologie Sociale (dirigé par C. Levi-Strauss), alors situés dans les sous-sols du Musée de l'Homme au Trocadéro, un colloque sur l'enseignement des Mathématiques et de la Statistique pour les Sciences Humaines, organisé par le Centre de Mathématique Sociale et de Statistique de l'E.P.H.E. - $6^{\text {e }}$ section (devenu depuis le Centre d'Analyse et de Mathématique Sociales de l'E.H.E.S.S.) que dirigeait G.-Th. Guilbaud. Y participèrent une cinquantaine d'enseignants et de chercheurs, venus de sept Universités de province et d'autant d'institutions parisiennes.

L'objet de cette rencontre était double!:

- Faire le point sur les enseignements de statistique, et même parfois de mathématiques autres que la statistique, dispensés dans certaines Facultés de Lettres et Sciences humaines, pour des étudiants de certaines spécialités (sociologie et psychologie essentiellement, linguistique ou philosophie parfois),

- Mettre en chantier la préparation de ce que pourraient être des enseignements d'algèbre générale, d'algèbre linéaire, de calcul des probabilités et de statistique pour ces disciplines, dans l'ensemble des universités françaises.

À l'issue de ces trois journées d'exposés et de débats, les participants avaient dégagé une doctrine claire!: pas d'enseignement de la statistique sans enseignement préalable d'un minimum d'algèbre linéaire, de combinatoire et de calcul des probabilités qui permettent à l'étudiant de comprendre ce qui, en statistique, n'était le plus souvent enseigné que sous forme de «!recettes!»!: recette du Chi-deux, par exemple, ou celle du «!T de Student!», etc.

Pour les études de psychologie et de sociologie, cette doctrine finit par triompher quatre ans plus tard, en 1966, lors de la réforme dite «!réforme Fouchet!» qui réorganisait les cursus!: création d'un premier cycle en deux ans (les DUEL et DUES, devenus depuis les DEUG), et d'une quatrième année, celle de maîtrise après la licence. 
Quelques temps après 1966, les géographes adoptaient le même point de vue!; comme certains des philosophes, non pour la statistique, mais pour l'enseignement de la logique pour lequel des préalables d'algèbre sont essentiels.

De 1962 à 1966, il fallut mener toute une action d'information et de formation des enseignants!; de nombreux stages spécialisés ou des cours hebdomadaires furent ainsi organisés. Mais l'existence d'un organe de liaison et d'information mutuelle entre les enseignants parut, aux participants du colloque, être un complément indispensable à ces actions. C'est ainsi que, lors de la dernière séance, fut prise à l'unanimité, la décision de créer «!Mathématiques et Sciences humaines!»!; par conséquent, dans les premières années, le contenu de la revue fut essentiellement pédagogique.

Parmi ces membres fondateurs du 16 mai 1962, certains sont depuis décédés, tels L. Frey, G. Noizet, R. Daval, F. Bresson, M. Thionet. D'autres sont encore heureusement parmi nous, au premier rang desquels G.-Th. Guilbaud, mais aussi parmi bien d'autres, C. Flament, P. Jullien, J.-F. Richard, M. Eytan, J. Leplat, B. Matalon, F.!Latreille, G. Morlat, M. Petruszewycz, P. Rosenstiehl, A. Rygaloff, M.C. Weiss. Cohorte à laquelle devaient, dès les mois suivants, se joindre des amis tels que A.!Degenne, E. Coumet ( $\dagger)$, H. Rouanet, R. Martin (†), ou G. Kreweras ( $\dagger$ )!; et bien sûr sur Charlotte Carcassone $(\dagger)$ qui fut pendant plus de trente ans la cheville ouvrière de la revue.

Et maintenant!? Un vaste renouvellement et rajeunissement du Comité de rédaction vient d'être opéré, et sera effectif à partir du premier numéro de 2003 $\left(\mathrm{n}^{\circ} ! 161\right)$. D'autre part, la possibilité d'adjoindre à l'édition «!papier!» actuelle une édition «!on line!» va être étudiée, et pourrait aboutir à une réalisation d'ici un an environ.

Quant au contenu, qui est l'essentiel, les prochains numéros - tout en poursuivant la publication d'articles «!varia!», continueront le dossier «!Démographie Mathématique!» (ouvert dans le $n^{\circ} 159$ ), avec des textes d'H. Le Bras, D. Courgeau et E.!Brian!; ouvriront un nouveau «!dossier!» consacré à la géographie mathématique et à la statistique spatiale.

Plusieurs numéros thématiques sont par ailleurs en chantier (leurs responsables sont indiqués entre parenthèses)!:

- Combinatoire et recherche opérationnelle (O. Hudry)

- Théorie des types (M. Bourdeau et P. Boldini)

- Théories du choix social (O. Hudry et B. Monjardet)

- Réseaux sociaux (A. Degenne)

- Mathématiques, jeux et sport (P. Parlebas)

- Place et rôle de l'informatique (J.-P. Desclés)

On le voit par la variété de ces domaines, la revue entend rester résolument généraliste tant en ce qui concerne les outils et méthodes mathématiques, que les sciences sociales!: nous ne nous adressons à aucune en particulier, car nous sommes toujours convaincus, comme dans les «!années 60 !», que chacune peut tirer profit de ce qui se fait chez les autres.

M.B. 